Original

\title{
Carcinoma de células renales con extensión a vena cava: puesta al día y revisión de nuestra casuística
}

\author{
Fernando Vázquez Alonso, Francisco J. Vicente de Prados, José M. Cózar Olmo, \\ Manrique Pascual Geler, Francisco J. Rodríguez Herrera, Antonio Martínez Morcillo, \\ Eduardo Espejo Maldonado, Miguel Tallada Buñuel
}

Servicio de Urología. Hospital Universitario Virgen de las Nieves. Granada, España

\section{Resumen}

Objetivo: Valorar la situación actual en el tratamiento del carcinoma renal con extensión a vena cava inferior (VCI): Clasificación, diagnóstico, abordaje quirúrgico, terapia adyuvante, factores de pronóstico y supervivencia.

Materiales y Métodos: Se revisan 19 casos de carcinomas renales con extensión a VCI intervenidos entre enero de 1988 y agosto de 2008. Se valoran la edad, el sexo, lateralidad y función renal de los pacientes. Con respecto al tumor se valoran: estadio según TNM y la clasificación de Neves-Zincke. Se describe el abordaje quirúrgico según el nivel del trombo.

Resultados: Con una tasa de mortalidad perioperatoria del 10.5\% y una media de seguimiento de 22,65 meses (rango 279), sobreviven 5 pacientes; 11 han fallecido por la enfermedad; uno por otra causa y 2 se han perdido. Los pacientes metastásicos han recibido tratamiento adyuvante con Inmunoterapia o inhibidores de las kinasas. La supervivencia media es de 15,1 meses. Existen diferencias significativas a tres y cinco años en la supervivencia de los pacientes estadiados como NOMO vs resto ( + +MO, NOM+, N+M+). No hay diferencias en función del nivel del trombo.

Conclusiones: El carcinoma renal con trombo en VCI es un tumor con alta mortalidad. El abordaje quirúrgico del mismo es la opción más valida y precisa una correcta valoración prequirúrgica y el apoyo de un equipo multidisciplinar preparado y con experiencia. La supervivencia depende de la extensión de la enfermedad.

Palabras clave: Tumor renal. Trombo en cava. Circulación extracorpórea. Factores pronósticos. Supervivencia.

\section{Renal cell carcinoma with vena cava involvement: update and review of our series}

\begin{abstract} diagnosis, surgical approach, adjuvant therapy, prognostic factors and survival rate. depended on thrombus level. stages ( $\mathrm{N}+\mathrm{MO}, \mathrm{NOM}+, \mathrm{N}+\mathrm{M}+$ ). No differences were found depending on thrombus level. Survival depends on disease extension.

Keywords: Renal tumor. Cava thrombus. Extracorporeal circulation. Prognostic factors. Survival rates.
\end{abstract}

Objective: To assess current management of renal cell carcinoma (RCC) extending into the inferior vena cava (IVC): staging,

Materials and Methods: Nineteen cases of RCC extending into the IVC undergoing surgical resection from January 1988 to August 2008 were reviewed. TNM staging and Neves-Zincke grading of the tumor were also assessed. Surgical approach

Results: With a perioperative mortality rate of $10.5 \%$ and a mean follow-up of 22.65 months (range 2-79), 5 patients are still alive, while 11 patients died from the disease, 1 from an unrelated cause, and 2 were lost to follow-up. Patients with metastatic disease received adjuvant treatment with immunotherapy or kinase inhibitors. Mean survival was 15.1 months. Significant differences were found in 3- and 5-year survival rates in patients staged as NOMO as compared to all other

Conclusions: RCC with thrombus in the IVC is a tumor with a high mortality rate. Surgery continues to be the best option, and requires adequate preoperative evaluation and the support of an experienced and well trained multidisciplinary team.

$\mathrm{E}$ 1 carcinoma de células renales representa el $2 \%$ de todos los cánceres del adulto ${ }^{1}$. Una de las características que presenta el carcinoma renal es su venotropismo; de hecho se estima que en el momento del diagnóstico es posible encontrar trombo tumoral en la vena renal en el 20-35\% de los casos, y extensión a la vena cava inferior (VCI) en el 4-10\%, pudiendo extenderse el trombo por encima de las venas suprahepáticas hasta alcanzar la aurícula derecha ${ }^{2-4}$. Cuando ocurre, la cirugía ofrece la única solución curativa ${ }^{5}$, siendo en estos casos compleja y requiriendo un amplio conocimiento de la anatomía y la participación de un equipo multidisciplinar. La vía de abordaje así como la técnica quirúrgica dependen del nivel que alcance el extremo cefálico del trombo tumoral en la vena cava. 
Además del carcinoma de células renales existen otras entidades que pueden originar un trombo tumoral en la vena cava, como pueden ser el carcinoma suprarrenal, carcinoma de pelvis renal, leiomiosarcoma de partes blandas, hepatocarcinoma, tumor de ovario y testículo, feocromocitoma, tumor de Wilms y el angiomiolipoma6-11. En nuestro Servicio fue intervenida una paciente con trombo en vena cava secundario a metástasis por cáncer de colon.

Presentamos nuestra experiencia quirúrgica en los últimos 20 años en pacientes con carcinoma renal con trombo tumoral en vena cava.

\section{MATERIAL Y MÉTODOS}

Entre enero de 1988 y agosto de 2008, 298 pacientes diagnosticados de carcinoma de células renales fueron sometidos a intervención quirúrgica en nuestro Servicio. De ellos 18 (6,04\%) presentaban en el momento de la cirugía trombosis venosa con extensión a VCI. Otro paciente tuvo trombosis tumoral en VCI a los 6,5 años de la nefrectomía radical por tumor renal.

Al diagnóstico, la edad media de los pacientes con trombo en cava fue de 69,3 años (34-76). La relación hombre: mujer fue de 5:1 (16 y 3). En cuanto a la lateralidad todos fueron derechos. La función renal era normal en 15 pacientes; 4 presentaban una insuficiencia renal crónica moderada-grave.

Para la estadificación de los pacientes donde existía extensión a VCI se utilizó la clasificación TNM y la serie se distribuía de la siguiente manera: 14 pacientes eran tipo T3b (73,6\%) y 5 eran T3c $(26,4 \%)$. El estadio patológico se resume en la Tabla 1.

Tabla 1. Estadio patológico

\begin{tabular}{lcc}
\hline & Frecuencia & Porcentaje \\
\hline pT3bNOM0 & 5 & 27,8 \\
pT3bNOM1 & 1 & 5,6 \\
pT3bN1M0 & 1 & 5,6 \\
pT3bN2M0 & 2 & 11,1 \\
pT3bN2M1 & 1 & 5,6 \\
pT3bNxM0 & 1 & 5,6 \\
pT3bNxM1 & 2 & 11,1 \\
pT3cNOM0 & 3 & 16,7 \\
pT3cNOM1 & 1 & 5,6 \\
T3bNOM1 & 1 & 5,6 \\
Total & 18 & 100,0
\end{tabular}

Si utilizamos la Clasificación de Neves-Zincke en donde se establecen 4 niveles (nivel I: renal; nivel II: infrahepático; nivel III: retrohepático; nivel IV: atrial), tres pacientes eran nivel IV, dos nivel III y catorce nivel IV.

Respecto a la estadificación ganglionar y metastásica en el momento del diagnóstico, 13 pacientes eran NO y 5 presentaban adenopatías en las pruebas de imagen. 5 pacientes eran metastásicos (4 pulmonares y 1 ósea/hepática).

La estrategia quirúrgica utilizada fue:

En los pacientes estadificados como T3b: en todos se practicó nefrectomía radical y trombectomía. En 3 casos fue necesaria la cavectomía segmentaria y ligadura de la vena renal izquierda. En todos los casos se realiza linfadenectomía de estadiaje.

En los pacientes estadiados como T3c: en 2 se llevó a cabo la nefrectomía radical y extracción del trombo tumoral con circulación extracorpórea (CE). En 2 casos no fue necesaria la CE y en el caso de recidiva tumoral se hizo sólo trombectomía con CE.

\section{RESULTADOS}

La mortalidad perioperatoria fue del 10,5\%. Un paciente falleció en el acto quirúrgico por embolismo pulmonar masivo al inicio de la intervención. Otro fallecimiento se produjo en el postoperatorio inmediato por fallo multiorgánico en la UCI (paciente con recidiva de cáncer renal). El resto evolucionaron de forma satisfactoria.

Con un seguimiento medio de 22,65 meses (279), en la actualidad sobreviven 5 pacientes; 11 han fallecido por la enfermedad; uno ha muerto por otra causa y 2 se han perdido para seguimiento.

De nuestra serie de pacientes, 4 (21\%) se trataron inicialmente con inmunoterapia, 2 (10,5\%) con sunitinib y en el resto se optó por vigilancia. De los 5 pacientes que sobreviven 2 viven sanos sin enfermedad y sin tratamiento adyuvante; uno desarrolló metástasis pulmonares a los 19 meses de la cirugía y tras progresión a pesar de inmunoterapia, en la actualidad recibe tratamiento con sorafenib con una supervivencia de 53 meses tras el diagnóstico de las metástasis pulmonares. Los otros dos viven con enfermedad metastásica desde el diagnóstico en tratamiento actual con sunitinib.

En los pacientes que han fallecido por la enfermedad la supervivencia media hasta la muerte es de 15,1 meses. 
Realizando un análisis de supervivencia mediante test de Kaplan-Meyer, comparando NOMO vs resto $(\mathrm{N}+\mathrm{MO}, \mathrm{NOM}+, \mathrm{N}+\mathrm{M}+)$ obtenemos unas diferencias estadísticamente significativas a tres y cinco años. De manera que los que no presentan lesiones a distancia (NOMO) tienen una supervivencia a los cinco años del $56,2 \%$, mientras que el resto, es decir, aquellos que presentan algún tipo de lesión a distancia presentan una supervivencia a los cinco años del 27\% (Fig. 1).

No hemos encontrado diferencias estadísticamente significativas en la supervivencia según el nivel del trombo (Fig. 2), aunque hay que tener en cuenta el bajo número de casos.

\section{DISCUSIÓN}

El cáncer renal con trombo en cava es uno de los mayores desafíos a los que se puede presentar un urólogo por su complejidad en el manejo quirúrgi$\mathrm{co}^{12}$. La excisión completa del trombo junto con la nefrectomía radical es la única opción de cura cuando se trata de pacientes sin afectación ganglionar ni metástasis a distancia ${ }^{5,7,13-15}$. El factor más importante a tener en cuenta para que la cirugía sea exitosa es el correcto manejo de la $\mathrm{VCI}^{16}$, siendo fundamental minimizar el sangrado y la prevención de embolismos a partir del trombo durante la cirugía, situaciones que cuando aparecen pueden llevar a consecuencias fatales ${ }^{17}$.

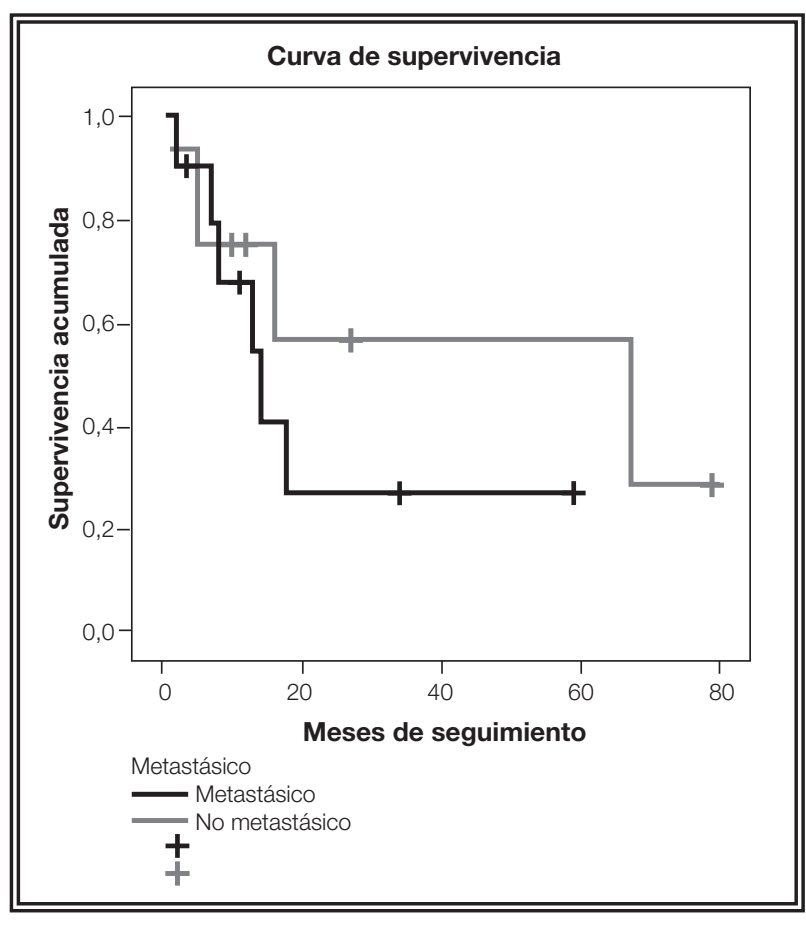

FIGURA 1. Supervivencia global NOMO vs Resto.

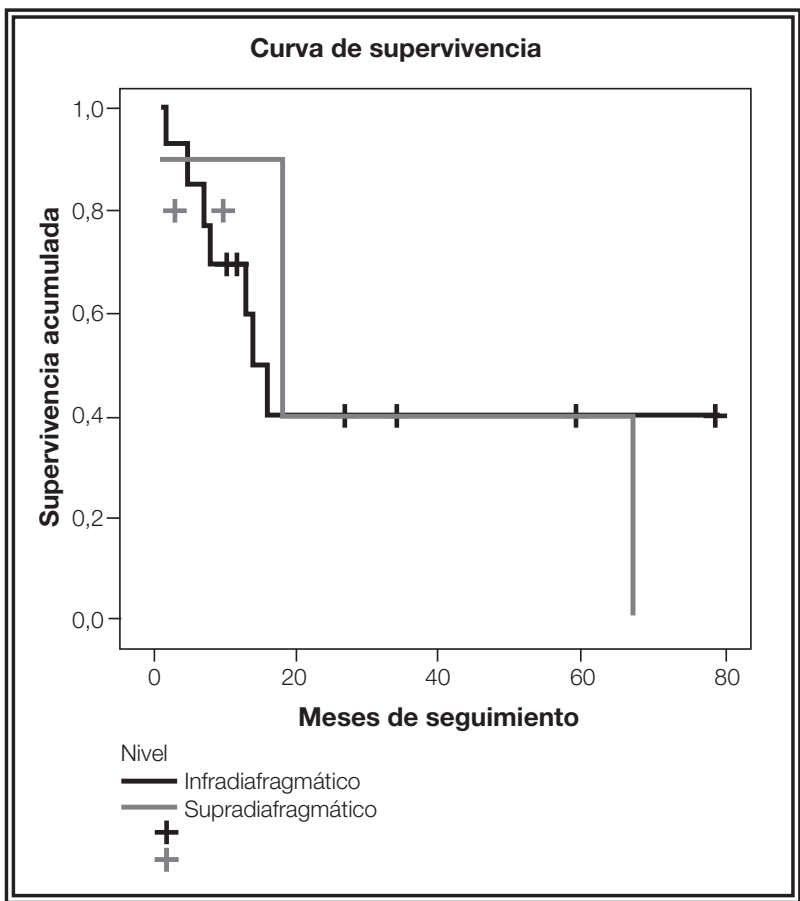

FIGURA 2. Supervivencia global según nivel del trombo.

Históricamente la presencia de un trombo venoso se ha asociado a un peor pronóstico en el carcinoma de células renales ${ }^{18,19}$, aunque varias investigaciones han señalado que la extensión vascular per se no es indicativo de una mayor agresividad por parte del tumor ${ }^{4}$ El valor pronóstico que tiene la altura del trombo es controvertido con resultados dispares publicados en diferentes series, tanto a favor $^{12,13,20}$ como en contra ${ }^{7,15,18,22}$. En la serie que aporta la Universidad de Miami encuentran un mayor índice de desarrollo metastásico en trombos de localización atrial en comparación con el resto de localizaciones ${ }^{17}$. No obstante hay autores que proponen que la relación entre el nivel del trombo y el pronóstico pueda estar relacionada con la dificultad en la técnica quirúrgica, con posibles extracciones incompletas del trombo en niveles altos ${ }^{12,23}$.

Sí es fundamental determinar el nivel cefálico del trombo para planificar la estrategia quirúrgica más adecuada en cada caso ${ }^{18,24,25}$. Para ello puede ser útil la utilización de la ecografía-doppler, ecografía transesofágica o la resonancia nuclear magnética con gadolinio ${ }^{24}$, siendo útil el TAC para el diagnóstico inicial; de entre estas pruebas diagnósticas se ha considerado la RNM como la más sensible y específica para la valoración de la presencia y extensión del trombo en los vasos representando el gold standard $^{26}$. 
La extensión craneal del tumor fue definida por Neves y Zincke ${ }^{27}$; se pueden establecer cuatro niveles: nivel I, renal; nivel II, infrahepático; nivel III, retrohepático; nivel IV, atrial. Esta clasificación define mejor la situación del trombo que la TNM, que únicamente diferencia dos apartados: T3b cuando el trombo se encuentra en la vena renal o en la cava por debajo del diafragma y T3c cuando el tumor se extiende por encima del diafragma ${ }^{25}$. Además hay autores que proponen modificar los estadios pT3b y pT3c en la próxima revisión de la TNM debido a las posibles diferencias en el significado pronóstico entre el trombo en vena renal y en $\mathrm{VCI}^{28}$.

En cuanto al pronóstico, parece que la existencia de trombo venoso se asocia a una menor supervivencia $^{19}$; sin embargo, es cierto que cuando existe trombo tumoral habitualmente el grado y estadio son más altos y las metástasis linfáticas y a distancia son dos veces más frecuentes. Los grados altos de la clasificación de Fuhrman, la presencia de ganglios positivos, la invasión de órganos vecinos o la coexistencia con metástasis a distancia ensombrecen notablemente la supervivencia de estos enfermos, más que la presencia y extensión del trombo $^{25,29}$. La infiltración tumoral de la vena cava se ha descrito como un factor pronóstico desfavorable ${ }^{20,26}$. Además, este hecho es importante tenerlo en cuenta por la posibilidad de llevar a márgenes quirúrgicos positivos. No obstante, existen grandes dificultades para definir preoperatoriamente la infiltración de la pared de la vena cava por el trombo tumoral. Recientemente está cobrando importancia la medición del diámetro anteroposterior de la VCI y del ostium de la vena renal mediante pruebas de imagen para predecir la infiltración venosa tumoral; así hay autores que plantean que un diámetro anteroposterior de la VCI mayor de $18 \mathrm{~mm}$ o del ostium de la vena renal mayor de $14 \mathrm{~mm}$ predicen con un $90 \%$ de sensibilidad la invasión tumoral de la $\mathrm{VCI}^{30}$.

En cuanto a la aparición de ganglios linfáticos positivos y/o metástasis a distancia, la mayoría de los autores están de acuerdo en que empeora significativamente la supervivencia ${ }^{31}$; en estos casos la cirugía puede estar justificada como actitud paliativa mejorando la calidad de vida o para reducir la carga tumoral previo a un tratamiento complementario $^{12}$; hasta hace poco se ha utilizado la inmunoterapia $^{15,32,33}$, pero en los últimos años está cobrando importancia el uso de los inhibidores multiquinasas como el sunitinib o sorafenib en el tratamien- to de este tipo de pacientes; incluso se han llegado a utilizar con éxito de forma neoadyuvante en determinados casos para reducir el trombo tumoral ${ }^{34}$.

Dos de los objetivos a controlar durante la cirugía son minimizar el sangrado y prevenir el embolismo del trombo, situaciones que si ocurren pueden llevar a consecuencias nefastas ${ }^{17}$.

Hay consenso en la literatura en la necesidad de trabajo con equipos multidisciplinarios si se trata de trombos de nivel III y IV, en cuyo caso debería recurrirse a centros de referencia que dispongan de equipos experimentados en cirugía del trasplante hepático para la movilización del hígado (Fig. 3) y la práctica de maniobras como pueda ser la maniobra de Pringle (oclusión temporal de la vena porta y del flujo arterial del hígado) y de cirugía cardiovascular, para la práctica de bypass cardiopulmonar con hipotermia en parada cardiaca, o la de bypass veno-venoso. Es interesante resaltar el papel que pueden jugar los dispositivos de recuperación de sangre en el campo operatorio, para evitar las pérdidas sanguíneas de condicionen la necesidad de politransfusión ${ }^{25}$.

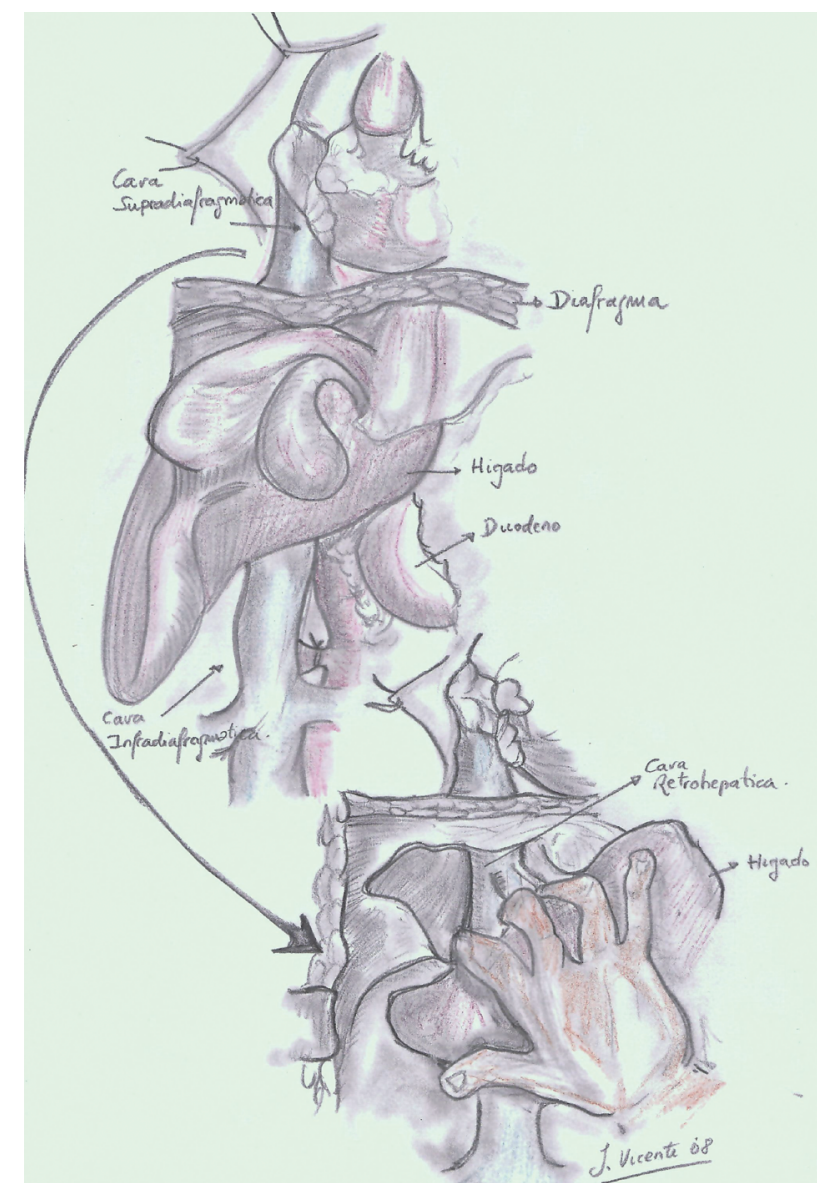

FIGURA 3. Acceso a la vena cava retrohepática. 
Existen controversias en cuanto a la selección de la estrategia adecuada, y en general, el bypass cardiopulmonar sólo debe plantearse con indicaciones muy precisas.

En determinados casos se podría plantear la utilización de arteriografía para evaluar el grado de arterialización del trombo que si es evidente, puede justificar la embolización arterial preoperatoria facilitando la intervención quirúrgica, ya que reduce la pérdida sanguínea mediante el colapso de los vasos colaterales en la superficie renal y en el hilio, y permite la ligadura de la vena renal sin tener que ligar primero la arteria; además, transcurridas 24-48 horas del infarto se desarrolla un edema considerable alrededor del tumor que facilita la disección de las estructuras contiguas ${ }^{17}$. Las complicaciones asociadas a este procedimiento incluyen la migración de los coils, la embolización incompleta o la formación de un hematoma inguinal en el 5\%; en el $74,4 \%-92 \%$ de los casos se producen una serie de síntomas como son dolor en el flanco, náuseas o vómitos de una intensidad moderada y autolimita$\operatorname{dos}^{35,36}$. Por tanto se puede considerar este procedimiento como seguro. No obstante, nosotros no hemos embolizado preoperatoriamente a ninguno de nuestros pacientes. En nuestra opinión, la ligadura precoz de la arteria renal permite encontrar pocas venas varicosas y escasa oposición a la disección del riñón; la circulación colateral queda rápidamente colapsada haciendo el resto de la disección más fácil, y tiene los mismos efectos que la embolización preoperatoria sin su morbilidad ${ }^{37}$, por lo que habría que reservar este procedimiento para casos muy seleccionados de especial complejidad.

En caso de invasión de la pared de la vena cava será necesario resecar algún segmento de su pared, total o parcialmente ${ }^{38}$. La sutura de la cavotomía debe permitir una luz venosa suficiente para el drenaje del riñón contralateral ${ }^{7}$, existiendo distintos planteamientos para la solución de este problema. Son útiles los parches con pericardio bovino liofilizado (Fig. 4). Puede practicarse la sustitución con Dacron o Goretex o ligar los extremos de la cava, (sobre todo si existe presencia de trombo blando infrarrenal) y de la vena renal izquierda; dicha situación será tolerada postoperatoriamente dependiendo del grado de oclusión preoperatoria de la cava $^{25}$.

El uso de filtros venosos para evitar la siembra tumoral y la embolización pulmonar, defendida por

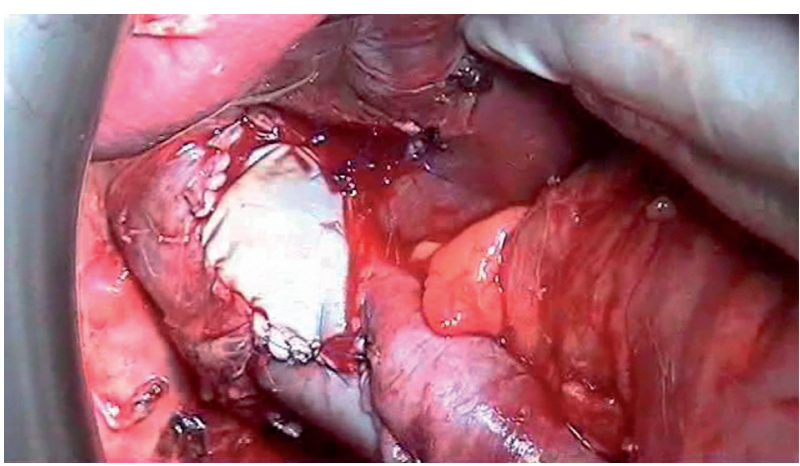

FIGURA 4. Parche de pericardio bovino sobre vena cava.

algunos autores ${ }^{39}$ es controvertido. Su colocación resulta un gesto quirúrgico sencillo y mínimamente invasivo, pero puede dificultar enormemente la cirugía a la hora de su extracción por lo que se debería evitar su uso siempre que fuera posible ${ }^{40}$. Su utilización es poco frecuente si se ha diseñado una correcta estrategia quirúrgica ${ }^{4}$.

Aunque la cirugía abierta es el gold standard en este tipo de tumores, existen centros con amplia experiencia en laparoscopia que han comunicado pequeñas series de pacientes con trombos en cava próximos a vena renal, sometidos a nefrectomía con trombectomía laparoscópica con buenos resultados a corto plazo, aunque son necesarios más estudios para examinar las ventajas de este procedimiento con respecto a la cirugía abierta ${ }^{41,42}$.

\section{REFERENCIAS}

1. Landis SH, Murria T, Bolden S, Wingo PA. Cancer statistics. CA Cancer J Clin 1999;49(1):8-31.

2. Skinner DG, Colvin RB, Vermillion CD, Pfister RC, Leadbetter WF Diagnosis and management of renal cell+carcinoma. A clinical and pathologic study of 309 cases. Cancer. 1971;28(5): 1165-1177.

3. Marshall FF, Dietrick DD, Baumgartner WA, Reitz BA. Surgical management of renal cell carcinoma with intracaval neoplasic extension above the hepatic veins. J Urol. 1988;139(6):1166-1172.

4. Garcia OD, Fernández Fernández E, de Vicente E, Honrubia A, Moya JL, Abella V, et al. Estrategia quirúrgica en el carcinoma de células renales con extensión a la circulación venosa. Actas Urol Esp. 2005;29(5):448-456.

5. Tribble CG, Gerkin TM, Flanagan TL, Pitman JM, Kron IL. Vena caval involvement with renal tumours: surgical considerations. Ann Thorac Surg. 1988;46(1):36-39.

6. Ortiz Gorráiz M, Tallada Buñuel M, Vicente Prados FJ, Rodríguez Herrera F, Rosales Leal JL, Honrubia Vílchez B, et al. Carcinoma suprarrenal izquierdo con trombo en cava. Arch Esp Urol 2003;56(5):485-489.

7. Rioja Zuazu J, Rodríguez-Rubio Cortadellas F, Zudaire Bergera JJ, Saiz Sansi A, Rosell Costa D, Robles García JE, et al. Cirugía con circulación extracorpórea e hipotermia en tumores con extensión a vena cava: 20 años de experiencia de la Clínica Universitaria de Navarra. Actas Urol Esp. 2008;32(4):396-405.

8. Quicios Dorado C, Allona Almagro A. Angiomiolipoma renal causante de trombo en cava y Síndrome de Budd-Chiari secundario. Arch Esp Urol. 2008:61(3):435-439. 
9. López Ferrandis J, Saiz Sansi A, Cervera Delgado M, Rioja Zuazu J, Regojo Balboa JM, Berián Polo JM. Feocromocitoma con trombo en cava. Actas Urol Esp. 2005;29(3):281-286.

10. De Castro F, Agüera LG, Robles JE, Llorens R, Abad JI, Rosell D, et al. Leimiosarcoma de la vena cava: Presentación de dos casos clínicos. Actas Urol Esp. 1993;17(8):504-507.

11. De Castro F, Robles JE, Zudaire JJ et al. Tratamiento quirúrgico de la trombosis tumoral de la vena cava inferior. Rev Cir Ibero-Am. 1:254, 1992.

12. Kirkali $Z$, Van Poppel H. A critical analysis of surgery for kidney cancer with vena cava invasion. Eur Urol. 2007;52(3):658-662.

13. Haferkamp A, Bastian PJ, Jakobi H, Pritsch M, Pfitzenmaier J, Albers P, et al. Renal cell carcinoma with tumor thrombus extension into the vena cava: prospective long-term folloup. $J$ Urol. 2007; 177(5): 1703-1708.

14. Taweemonkongsap T, Nualyong C, Leewangsangtong S, Amornvesukit T, Sirivatanauksorn Y, Tantiwong A, et al. Surgical treatment of renal cell carcinoma with inferior vena cava thrombus: using liver mobilization technique to avoid cardiopulmonary bypass. Asian J Surg. 2008;31(2):75-82.

15. Parra OR, Gómez Jiménez J, Gilson PM. Experiencia en el tratamiento del carcinoma de células renales con trombo en vena cava inferior y aurícula derecha. Actas Urol Esp. 1997;21(6):550-557.

16. Swierzewski DJ, Swierzewski MJ, Libertino JA. Radical nephrectomy in patients with renal cell carcinoma with venous, vena caval and atrial extension. Am J Surg. 1994;168(2):205-209.

17. Ciancio G, Livingstone AS, Soloway M. Surgical management of renal cell carcinoma with tumor thrombus in the renal and inferior vena cava: the University of Miami experience in using liver transplantation techniques. Eur Urol. 2007;51(4):988-995.

18. Ruibal Moldes M, Álvarez Costelo L, Chantada Abal V, Blanco Díez A, Fernández Rosado E, González Martín M. Cirugía del carcinoma renal con trombo tumoral en vena cava-aurícula. Actas Urol Esp. 2003;27(7):517-523.

19. Ortiz Gorráiz M, Vicente Prados FJ, Rosales Leal JL, Honrubia Vílchez B, Martínez Morcillo A, Cózar Olmo JM, et al. Valoración de factores pronósticos de la supervivencia en una serie de 202 pacientes intervenidos por carcinoma de células renales. Actas Urol Esp. 2005;29(2):179-189

20. Sosa RE, Muecke EC, Vaughan ED Jr, McCarron JP Jr. Renal cell carcinoma extending into the inferior vena cava: the prognostic significance of the level of vena caval involvement. $J$ Urol. 1984;132(6): 1097-1100.

21. Sweeney P, Wood CG, Pisters LL, Slaton JW, Vaporciyan A, Munsell $\mathrm{M}$, et al. Surgical management of renal cell carcinoma associated with complex inferior vena caval thrombi. Urol Oncol. 2003;21(5): 327-333.

22. Bissada NK, Yakout HH, Babanouri A, Elsalamony T, Fahmy W, Gunham $\mathrm{M}$ et al. Lomg-term experience with management of renal cell carcinoma involving the inferior vena cava. Urology. 2001;61(1): 89-92.

23. Hatcher PA, Anderson EE, Paulson DF, Carson CC, Robertson JE Surgical management and prognosis of renal cell carcinoma invading the vena cava. Ann Vasc Surg. 2005;19(4):522-528.

24. Karnes RJ, Blute ML. Surgery insight: management of renal cell carcinoma with associated inferior vena cava thrombus. Nat Clin Pract Urol. 2008;5(6):329-339.

25. Tallada Buñuel M, Miñana López B. Tratamiento quirúrgico. Actualización de las opciones terapéuticas médico-quirúrgicas en el carcinoma renal avanzado y metastásico. LXXIII Congreso Nacional de Urología. Barcelona, 2008.

26. Lawrentschuk N, Gani J, Riordan R, Esler S, Bolton DM. Multidetector computed tomography vs magnetic resonante imaging for defining the upper limit of tumour trhrombus in renal cell carcinoma: A study and review. BJU Int 2005;96(3):291-295.

27. Neves RJ, Zincke H. Surgical treatment of renal cancer with vena cava extension. Br J Urol. 1987;59(5):390-395
28. Wagner B, Patard JJ, Méjean A, Bensalah K, Verhoest G, Zigeuner $\mathrm{R}$, et al. Prognostic value of renal vein and inferior vena cava involvement in renal cell carcinoma. Eur Urol 2008 Aug 5.

29. Ljungberg B, Hanbury DC, Kuczyk MA, Merseburger AS, Mulders $\mathrm{PF}$, Patard JJ, et al. Renal cell carcinoma guideline. Eur Urol. 2007;51(6): 1502-1510

30. Zini L, Destrieux-Garnier L, Leroy X, Villers A, Haulon S, Lemaitre $\mathrm{L}$, et al. Renal vein ostium wall invasion of renal cell carcinoma with an inferior vena cava tumor thrombus: prediction by renal and vena caval vein diameters and prognostic significance. J Urol. 2008;179 (2):450-454.

31. Tanaka M, Fujimoto K, Okajima E, Tanaka N, Yoshida K, Hirao Y. Prognostic factors of renal cell carcinoma with extension into inferior vena cava. Int J Urol. 2008;15(5):394-398.

32. Parekh DJ, Cookson MS, Chapman W, Harrell F Jr, Wells N, Chang $\mathrm{SS}$, et al. Renal cell carcinoma with renal vein and inferior vena caval involvement: clinicopathological features, surgical techniques and outcomes. J Urol. 2005;173(6):1897-1902.

33. Kato T, Madono K, Saito J, Kakuta Y, Tanigawa G, Yazawa K, et al. Successful preoperative interferon-alfa therapy of advanced renal cell carcinoma with tumor thrombus extending into the inferior vena cava: a case report. Hynyokika Kiyo. 2007;54(2):119-22.

34. Di Silverio F, Sciarra A, Parente U, Andrea A, Von Heland M, Panebianco V, et al. Neoadjuvant therapy with sorafenib in advanced renal cell carcinoma with vena cava extension submitted to radical nephrectomy. Urol Int. 2008;80(4):451-453.

35. Schwartz MJ, Smith EB, Trost DW, Vaughan ED Jr. Renal artery embolization: clinical indications and experience from over 100 cases. BJU Int. 2007;99(4):881-886.

36. Serafin Z, Karolkiewicz M, Strze冈niewski P, Lasek W, Bryczkowski M, Wolski Z. Palliative percutaneous kidney embolization with enbucrilate in patients with renal cell carcinoma: safety and symptom control. Med Sci Monit. 2007;13 Suppl 1:98-104.

37. Ciancio G, Vaidya A, Soloway M. Early ligation of the renal artery using the posterior approach: a basic surgical concept reinforced during resection of large hypervascular renal cell carcinoma with or without inferior vena cava thrombus. BJU Int. 2003;92(4):488-489.

38. Vicente Prados FJ, Tallada Buñuel M, Pastor J, Martínez Morcillo A, Cózar Olmo JM, Espejo Maldonado E, et al. Adenocarcinoma renal con invasión de vena cava: estado actual de su diagnóstico y tratamiento mediante cavectomía total segmentaria. Arch Esp Urol. 1998;51(1):35-41.

39. Blute ML, Boorjian SA, Leibovich BC, Lohse CM, Frank I, Karnes RJ. Results of inferior vena caval interruption by Greenfield filter, ligation or resection during radical nephrectomy and tumor thrombectomy. J Urol. 2007;178(2):440-445.

40. Juan Escudero JU, Ramos de Campos M, Cánovas López S, Fabuel Deltoro M, Marqués Vidal E. Tratamiento quirúrgico de carcinoma renal con trombo y filtro en vena cava. Arch Esp Urol. 2008;61(6):730733.

41. Varkarakis IM, Bhayani SB, Allaf ME, Inagaki T, Gonzalgo ML, Jarrett TW. Laparoscopic-assisted nephrectomy with inferior vena cava tumor thrombectomy: preliminary results. Urology. 2004;64(5): 925-929.

42. Martin GL, Castle EP, Martin AD, Desai PJ, Lallas CD, Ferrigni RG, et al. Outcomes of laparoscopic radical nephrectomy in the setting of vena caval and renal vein thrombus: seven-year experience. J Endourol. 2008;22(8):1681-1685.

Correspondencia autor: Dr. Fernando Vázquez Alonso Servicio de Urología. Hospital Universitario Virgen de las Nieves Avda. de las Fuerzas Armadas, 2 - 18014 Granada Tel.: 958020600

E-mail autor: fernando.vazquez.alonso.sspa@juntadeandalucia.es Información artículo: Original

Trabajo recibido: abril 2009

Trabajo aceptado: mayo 2009 